\title{
IUFOST2006/813 Nutrigenetic of fat-soluble microconstituents bioavailability
}

\author{
P. Borel \\ UMR INSERM / INRA 'Nutrition Humaine et Lipides', Faculté de Médecine, 27 boulevard Jean Moulin, \\ 13385 Marseille, France \\ patrick.borel@medecine.univ-mrs.fr
}

The absorption efficiency of fat-soluble microconstituents (FSM), i.e. vitamins A, E, D and K, carotenoids and phytosterols, is very variable and depends on numerous factors. Since most of these molecules have health benefits, a better knowledge on factors which affect their absorption/bioavailability is particularly important. It has long been assumed that most FSM are absorbed by a passive process. However, it has recently been shown that phytosterols are effluxed, i.e. pumped back to the intestinal lumen, by two membrane transporters (ABCG5 and ABCG8). Recent studies have also shown that membrane transporters are involved in carotenoids and vitamin E absorption. SR-BI is involved in apical uptake of vitamin E and carotenoids and ABCA1 is implicated in vitamin E secretion at the basolateral side of the enterocyte. It can be hypothesized that mutations or SNPs in one or several of these transporters may lead to modifications in their absorption efficiency. Our team will test this hypothesis in a European integrated project called "Lycocard". In this project, the absorption efficiency of lycopene will be related to polymorphisms (SNPs) in candidate transporters of this carotenoid. Personalized nutrition is likely the future of human nutrition. In fact, there is already a demand on personalized pharmacology. As a matter of fact an US company proposes to adjust the posology of drugs with regard to the genotype of subjects for enzymes of xenobiotic metabolism. It can be anticipated that the same kind of approach will be available in nutrition, peculiarly for FSM which can be beneficial at low doses and hazardous at higher doses. It is therefore reasonable to anticipate that doses of micronutrients in functional foods/supplements will be adapted to sub-groups carrying SNPs in genes known to affect FSM bioavailability. Knowledge on nutrigenetic of FSM bioavailability will allow food companies to state that the dose of FSM incorporated in their foods is not harmful, even for the higher responders to the micronutrient incorporated. It will also be possible to elaborate different doses of FSM for low and high responders. In summary the discovery that membrane transporters are involved in the absorption of FSM is a key finding in the field of FSM bioavailability. Interindividuals variations of expression and/or activity of these transporters probably explain the high interindividual variability in FSM absorption. The future knowledge in nutrigenetic of bioavailability will probably lead to more personalized recommendations that takes into account genetic characteristics of subgroups of the population. 